\title{
A Correlational Analysis of the Relationship between Perceived Peer Pressure and Decision Making in Adolescents
}

\section{Trisita Bhattacharyya ${ }^{1}$, Debasmita Roy ${ }^{1}$, Sunetra Chatterjee ${ }^{1}$, Sharwat A. Querashi ${ }^{1}$ and Tinni Dutta ${ }^{2 *}$}

\author{
1Department of Psychology, Asutosh College, University of Calcutta, Kolkata \\ 2Department of Psychology, Muralidhar Girls' College, University of Calcutta, Kolkata \\ E-mail: trisitab@gmail.com; reemaroy013@gmail.com ; sunetrachatterjee6@gmail.com; \\ sharwataafreenquerashi@gmail.com; tinnid@yahoo.com \\ *Corresponding author details: Dr. Tinni Dutta, tinnid@yahoo.com
}

\begin{abstract}
Adolescence is a stage of human development during which individuals are known to crave independence from authority figures and demonstrate an inclination to take their own decisions and solve their own problems. Since, research has shown that adolescents spend twice as much time with their peers than they do with their parents or other adults; we anticipated that a relationship is present between the peer pressure experienced by adolescents and their emerging decision making styles. Thus, this study was conducted, to explore the correlation between the decision making styles and perceived peer pressure of adolescents using a sample of 30 adolescents (17 males, 13 females), with ages varying from 14 to 17 years ( $m=16.43$ ). Data was collected using the 'Adolescent Decision Making Questionnaire', the 'Perceived Peer Pressure Scale' and a personal information form. It was observed that a significant positive correlation exists between the perceived peer pressure scores and the scores for panic, evasiveness and complacency patterns of decision making; implying that greater the peer pressure on a decision maker, greater is their inclination to resort to panic, evasive and complacent decision making styles.
\end{abstract}

Keywords: perceived peer pressure; decision making style; adolescents; panic; evasiveness; complacency

\section{INTRODUCTION}

Adolescence is the stage in the life-span development of an individual that marks the transition from puberty to adulthood, stretching from the age of 13 years until 18 or the age of legal maturity. It is considered to be a very important period of development, since not only does it have major immediate impact on the attitudes and behaviours of a person, but also has significant long term effects on the rest of their lives. During this period a person undergoes huge social, physical and psychological changes. The enormity of such changes usually cause adolescents to suffer from feelings of heightened emotionality and uncertainty regarding their capacities and abilities. The increasing responsibilities and changing social roles predispose them to feelings of insecurity and instability and they struggle to make sense of the problems and decisions which seem more difficult to cope with, than the ones that they have had to face before [10].
Another noticeable characteristic of this transition is marked by the tendency of the adolescents to gain independence from the influence of parents and other authority figures and form relationships on their own. They tend to spend more time with their peers than with the adults in their lives. Gradually, this peer group which consists of a small group of similar aged fairly close friends [12], becomes an integral source of emotional as well as social support for them. According to Dacey and Kenny [6], adolescents who feel accepted by their peer groups are likely to feel good about themselves. Peers are found to strongly determine preference in the way of dressing, speaking, using illicit substances, sexual behaviours, adopting and accepting violence, adopting criminal and anti-social behaviours and in many other areas of the adolescent's life [19]. 
Research has also shown that peer pressure, or the demands for compliance to customs of the group and dedication and loyalty to its members [15], has the greatest influence on adolescent behaviour and decision making of all other factors. It can either be positive or negative depending on what behaviours are adopted and what consequences they yield to. If the pressure to conform to the group expectations enriches the personality of an individual, it is positive. On the contrary, if the pressure gives rise to negative attitudes and unhealthy beliefs it is considered to be negative. Extreme pressures from the group can make an individual lose their individuality. Consequently, adolescents are often forced to adopt particular lifestyles as a result of pressure from the peer groups to which they belong [18]. Hence, in a nutshell, it can be said that peer pressure, plays an important role in shaping a person's interests, values and beliefs during the adolescent stage.

Now, decision making is a process where an individual makes a judgement based on the available alternatives. According to the conflict theory by Janis and Mann [11], there are 4 principal decision coping patterns that an individual may utilise to deal with decisional conflicts. Each such pattern or style of decision making is determined by the antecedent conditions, the time pressure, the optimism or pessimism about finding a solution and also the degree of psychological stress that the individual is experiencing. Vigilance is a style of decision making which is employed when the time pressure is low and the person is optimistic about finding a solution. It is characterised by a thorough search of the available alternatives and an objective evaluation of the decision before finalising it. The panic pattern of decision making, on the other hand is employed when both, the perceived time pressure and stress levels are of the individual are quite high. It leads to hastily contrived decisions taken to escape the stress of the situation. The evasive or defensive avoidance style is utilised when the person is pessimistic about finding a solution and tries to escape the conflict by procrastination, buck-passing or rationalisation. The fourth and last decision coping pattern discussed is complacency, wherein the individual exhibits unconflicted change. That is, he/she usually dismisses the conflict or threat and continues unchanged or reflexively adapt to whatever is offered in the situation.

However, since peer influence is strong in the case of adolescents, it becomes tough for them to resist peer pressure and hence adolescents are reported to often base their decisions on group norms preferring group acceptance above their own choices.

In the current study we have measured and defined the perceived peer pressure and the degree of preference for all the 4 reported decision making styles of 30 adolescents. It is also investigated whether there exists a relationship between these two variables.

\section{LITERATURE REVIEW}

- In 1993, Isaac A. Friedman and Leon Mann [8] compared the decision coping patterns of Israeli and Australian adolescents using two samples of 1028 Israeli and 428 Australian high-school students (age range 13.5-14.4 years). Both the samples were administered the
Adolescent Decision Making Questionnaire. Results showed that the Australian adolescents scored lower than Israeli adolescents on self-confidence and vigilance, but higher on evasive behaviour in decision making. In both the samples girls scored higher than boys on panicky behaviour, although boys outscored girls on self-confidence and on complacency. For both samples, it was established that decision coping patterns can be reduced to two distinct clusters- a vigilant strategy and maladaptive strategies (panic, evasion, and complacency).

- Brown, B. B. (1990) [3] In his paper "Peer groups and peer cultures" stated that high school students spend twice as much of their time with peers as with parents or other adults. He further studied the accounts for the emergence of peer groups and peer cultures in adolescence, the place of adults in adolescent peer groups and the ways in which peer groups affect individual behaviour and psychological well- being.

- V. Palani and S. Mani in their article titled "Exploratory Factor Analysis: Development of Perceived Peer Pressure Scale" [17], developed a scale to study higher secondary students; perceived peer pressure. The scale consists of 30 items, categorized under three dimensions. The dimensions are: Yielding to Peer Pressure, Resistance to Peer Pressure and Peers Encouragement. The reliability and validity value of the final tool indicated that the perceived peer pressure scale is valid for application.

- Using retrospective accounts from 297 college undergraduates, B. Bradford Brown's study on 'The extent and effects of peer pressure among high school students: A retrospective analysis' [2], assessed how much pressure peers exerted in numerous areas of high school life and how this pressure influenced teenagers' attitudes and behaviors. One-third of both genders identified peer pressure as one of the hardest things they had to face as a teenager. The findings suggested that peer pressure appeared stronger for females than males. Perceptions of peer pressure were significantly associated with dating attitudes, sexual activity, and use of drugs and alcohol, but not with relationships with parents.

- Aditi Acharya and Guptain [1] in 2014, conducted a research to study the influence of peer pressure on brand switching among Indian college students. The study aimed to identify the drivers of and barriers to brand switching among college students in India, and check for the influence of peer pressure on brand switching in the context of four product categories mobile network, ice cream, deodorant and movie theatre. A positive relationship was found between the peers using a brand or favoring a brand and the consumer switching to that brand. The survey responses additionally revealed that sharing whole of brand name of name opinions were correlative with brand switch, thereby reinforcing the strength of peer pressure.

- Peer pressure is consistently implicated in the excessive drinking of college students. Brian Borsari and Kate B. Carey [4] in 2001 in the paper 'Peer influences on college 
drinking: A review of the research found ample evidence that demonstrated the interpersonal processes influenced student drinking. Peer environment and pressure was found to contribute highly to high-risk alcohol use by ways of modelling and perceived social norms.

- Leon Mann, Ross Harmoni and Collin Power [14] submitted a review that focuses on cognitive aspects of decision making and discusses nine indicators of competence: choice; comprehension; creativity; compromise; consequentiality; correctness; credibility; consistency; and commitment. The evidence suggests that by the age of 15 years many adolescents show a reliable level of competence in metacognitive understanding of decision making, creative problem-solving, correctness of choice, and commitment to a course of action. Young adolescents (12-14 years) find it harder to create options, identify a wide range of risks and benefits, foresee the consequences of alternatives, and gauge the credibility of information from sources with vested interests. No available evidence explains and effect of age differences in willingness to make choices, devise compromises, and show consistency of choices.

- Susan Lane's study titled “Decision making behaviors for adolescent mothers" [13] attempted to explore decision making behaviors, stressors, and social support for teenage mothers. It was inferred that understanding how decision making occurs in this population of adolescents, is an initial step in prevention of adolescent pregnancy. Thus, prevention programs can be tailored to meet the decision-making needs of this population.

\section{OBJECTIVE}

To investigate the relationship between the perceived peer pressure and decision making styles of adolescents.

\section{SAMPLE CRITERIA}

A total of 30 participants were taken falling in the age group 14-18, namely adolescents. It was done on the basis of Purposive Sampling. Purposive sampling is intentional selection of informants based on their ability to elucidate a specific theme, concept, or phenomenon. A sample of 17 males (56.66\%) and 13 females (43.33\%) were selected for the study.

\section{TOOL}

The Adolescent Decision Making Questionnaire [14] is a self-report questionnaire consisting of 30 items. It measures self-confidence in decision making and the four decision making styles: vigilance (Cronbach's alpha = $0.73)$; panic (alpha $=0.70)$; evasiveness $($ alpha $=0.66)$ and complacency (alpha $=0.73$ ) [8]. The total number of items in each subscale are: self-confidence (6), vigilance (6), panic (4), evasiveness (6) and complacency (8). Respondents could mark them as Strongly Agree (scored 5), Agreed (scored 4), Neutral (scored 3), Disagreed (scored 2) and Strongly Disagreed (scored 1). A high score represents a high level of the respective subscale. There were three exceptional items (item nos. 2, 4 and 6) for the subscale Self Confidence which were reversed, because of their negative formulation.

The Peer Pressure Scale Questionnaire [17] was used in which participants answered a total of 30 questions (Cronbach's alpha $=0.942$ ) assessing school performance, substance abuse, theft as well as dating and sexual behaviour. Respondents marking was the same as in the previous scale following the Likert scale. Here the scores of the items under the factor "Resistance to peer pressure" were reversed due to their obvious negative formulations.

A personal information form was also administered to the participants. It mainly required them to provide information regarding their name, age, gender and educational qualification.

\section{PROCEDURE}

Following consent, a total of 30 participants were selected. They were sent online questionnaires in the form of google form, which they had to fill. There were namely two questionnaires, one on decision making and one on peer pressure. They had to read the statements and answer if they Strongly Agreed, Agreed, Strongly Disagreed, Disagreed or were Neutrally disposed towards them.

After the submission of the form, data was collected and necessary descriptive statistical calculations (Mean and Standard Deviation) and Pearson's Correlations were carried out for further discussion.

In this study parametric statistical methods were used for the analysis of the data. It was reasoned that in spite of the small size of the sample and Likert data, using parametric statistical methods will not affect the accuracy of the results since these statistical methods are found to be considerably robust towards the violation of such assumptions [16].

\section{SCORING}

Here, a scoring system has been established, following the Likert scale where Strongly Agree has a score of 5, Agreed has a score of 4, Neutral has a score of 3, Disagreed has a score of 2 and Strongly Disagree has a score of 1 . In some exceptional items (as mentioned earlier) the scoring was reversed.

\section{RESULTS}

The descriptive statistics for the data obtained in the Adolescent Decision Making Questionnaire (ADMQ) and Perceived Peer Pressure Scale (PPPS) are as follows:

TABLE 1: Descriptive statistics for ADMQ and PPPS

\begin{tabular}{|c|c|c|}
\hline $\begin{array}{c}\text { SCORES } \\
\text { OBTAINED }\end{array}$ & MEAN (m) & $\begin{array}{c}\text { STANDARD } \\
\text { DEVIATION } \\
\text { (SD) }\end{array}$ \\
\hline $\begin{array}{c}\text { SELF- } \\
\text { CONFIDENCE } \\
\text { (ADMQ) }\end{array}$ & 19.43 & 4.09 \\
\hline $\begin{array}{c}\text { VIGILANCE } \\
\text { (ADMQ) }\end{array}$ & 21.30 & 3.50 \\
\hline $\begin{array}{c}\text { PANIC (ADMQ) } \\
\text { EVASIVENESS } \\
\text { (ADMQ) }\end{array}$ & 13.46 & 3.46 \\
\hline
\end{tabular}




\begin{tabular}{|c|c|c|}
\hline $\begin{array}{c}\text { COMPLACENCY } \\
\text { (ADMQ) }\end{array}$ & 22.46 & 4.40 \\
\hline $\begin{array}{c}\text { PERCEIVED } \\
\text { PEER }\end{array}$ & 84.63 & 7.63 \\
$\begin{array}{c}\text { PRESSURE } \\
\text { (PPPS) }\end{array}$ & & \\
\hline
\end{tabular}

Thus, it can be observed that the standard deviation is maximum for the complacency decision making style and is minimum for the panic or hypervigilance pattern. This indicates that the variation in the responses received is greatest for complacency and the lowest for panic. It was also seen that the standard deviation for perceived peer pressure scores is higher than any of the decision making style included in the ADMQ, hence implying a greater dispersion of the responses.

In order to examine the relationship between the scores obtained by the adolescents in the perceived peer pressure Scale (PPPS) and the Adolescent Decision making Questionnaire (ADMQ), the Pearson's Correlation Coefficients were calculated. The results are as follows:

TABLE 2: Pearson's Correlation Coefficients between ADMQ and PPPS scores

\begin{tabular}{|c|c|c|c|c|c|}
\hline ADMQ & $\begin{array}{c}\text { SELF- } \\
\text { CONFID } \\
\text { ENCE }\end{array}$ & $\begin{array}{c}\text { VIGILA } \\
\text { NCE }\end{array}$ & $\begin{array}{c}\text { PANI } \\
\text { C }\end{array}$ & $\begin{array}{c}\text { EVASI } \\
\text { VENES } \\
\text { SPS }\end{array}$ & $\begin{array}{c}\text { COMPLA } \\
\text { CENCY }\end{array}$ \\
\hline $\begin{array}{c}\text { PERCE } \\
\text { IVED } \\
\text { PEER }\end{array}$ & -0.057 & -0.065 & 0.379 & 0.404 & 0.574 \\
$\begin{array}{c}\text { PRESS } \\
\text { URE }\end{array}$ & & & & & \\
\hline
\end{tabular}

Thus we can see that, significant positive correlations were observed between the peer pressure scores and the scores for panic $(r=0.379 ; \mathrm{p}<0.01)$, evasiveness ( $\mathrm{r}=0.404 ; \mathrm{p}<$ $0.05)$ and complacency $(r=0.574 ; \mathrm{p}<0.01)$. Negative correlation values were obtained between the peer pressure scores and the scores for vigilant decision making $(\mathrm{r}=-0.065 ; \mathrm{p}>0.05)$ and self-esteem $(\mathrm{r}=-0.057 ; \mathrm{p}>0.05)$ of the adolescents. However, the correlation values obtained in the last two case weren't significant and hence, would not bear much value in our interpretation of the data.

\section{DISCUSSION}

According to Friedman and Mann [8], 1993, the decision coping patterns identified by Janis and Mann in their conflict theory (1977) [11] can be classified into two categories: adaptive and maladaptive decision coping patterns. Adaptive decision making styles or patterns are usually known to involve carefully deliberated behaviour. The only decision making style considered in our study, which corresponds to this category is vigilant decision making. This style, characterised by a thorough search of all the available alternatives and an objective evaluation of the costs and benefits of the decision before finalising it, can be considered to be leading to the most well-adjusted decisions of an individual's life. Most of the items (especially nos. 8 and 16), under the vigilance category of the questionnaire, can be observed to have received a majority of affirmative responses $(73.33 \%$ and $86.66 \%$ respectively), with adolescents in deed agreeing to "taking a lot of care before making their choice" and "thinking before taking a decision". The negative insignificant correlation that is observed between the peer pressure scores and the scores for vigilance in this study, indicates that the greater the peer pressure on the decision maker lesser is the self-reported tendency of employing a vigilant decision making style. However, the insignificant nature of the correlation, renders this relationship to be negligible in value.

Among the maladaptive decision making styles, varied responses were recorded across the different categories. Items under panic or hypervigilance decision coping patterns, received a majority of affirmative or neutral responses, indicating the adolescents' self-reported tendency of making hasty decisions to escape from the stress of the situation. The category for evasiveness, however, is characterised mainly by negative or neutral responses. This can lead to the conclusion that the selfreported tendency of the adolescents in the sample to use defensive avoidance patterns of making decisions is considerably low. The maximum negation recorded are of the items $12(66.66 \%)$ and $14(66.66 \%)$ which asked the participants to indicate, whether "they would let some one other make a decision for them so that it won't be their problem" and whether "they prefer to leave their decision to others" respectively. The final maladaptive decision coping pattern studied was that of complacency which is said to be the pattern when an individual exhibits unconflicted adherence or unconflicted change (Friedman and Mann, 1993) [8]. Here the person is observed to reflexively adapt to whatever is being offered and not being much concerned with changing the situation. Most of the items under this category received negative responses from the participants. While a somewhat affirmative $(36.66 \%)$ or undecided $(43.33 \%)$ outlook was observed for item no. 10 where the adolescents agreed to "go along with the suggestions of others, when faced with a decision", a slight contradiction could be observed as the same participants disagreed (53.33\%) to "drifting into decisions without thinking about them" (item no. 26). These maladaptive styles of decision making are usually utilised when the individual believes that they do not have sufficient time to make a decision, are pessimistic about reaching a proper solution or are stressed out by the circumstances [8].

The significant positive correlations which were obtained between the peer pressure scores and the scores for panic, evasiveness and complacency means that greater the peer pressure on the decision maker, greater is the selfreported tendency of employing maladaptive decision making patterns. Thus, the increase in peer pressure was observed to be significantly related with an increase in the use of panic, evasiveness and complacency styles of decision coping among the sample adolescents. Maladaptive decision making styles, are those which fail to meet many of the requirements of high quality information processing [8], thus, leading to poorly thought out and 
hasty decisions or tendencies of procrastination and avoidance.

The negative insignificant correlation obtained between the self-confidence and peer pressure scores of the adolescents in the study, indicates that greater the peer pressure of the decision maker lower is their degree of selfreported confidence. This correlation though, being not significant in nature, holds only negligible consequence. Most items in the self-confidence category were graced with mainly positive or neutral responses with item 5 ("The decisions I make turn out well") recording absolutely no negative responses at all. In fact, the only items with a greater number of disagreements were the items which had negative statement formulations. This can be considered to indicate a high degree of confidence among all the adolescents.

Regarding the perceived peer pressure scores of the adolescents, we can observe that the responses were varied at best. The category 'yielding to peer pressure' mostly garnered negative responses with maximum disagreements (83.33\%) being recorded by item no. 15 ("I would like to have an iPod because my friends expect me to have one"), and most positive (93.33\%) responses being given to item 28 ("when others make fun of my friends, I ought to defend my friends"). This demonstrates that though the participants have outgrown their mindless conformity of gang approved standards, which is a characteristic of late childhood [5], they do have feelings of solidarity for their peers. In the second category, 'resistance to peer pressure', item 20 ("I do not allow my friends to copy from my home assignments and test related activities") recorded a large number of negative responses (80\%), once again demonstrating the harmony, that exists among the adolescent participants of the study, for their close friends. In the final category, 'peer encouragement' which dealt with the positive effects of peer pressure a majority of positive responses were recorded implying that the individuals did receive "motivation to study" (item no. 26) (80\%) and "encouragement to solve difficult issues" (item no. 22) (76.66\%) from their friends. Contrary to the trend however, some amount of disagreement $(43.33 \%)$ was recorded in response to item no. 25 ("I joined swimming and other lifesaving training programmes, because of my friends' encouragement") indicating that while adolescents did benefit from their peers' encouragement in primary arenas of their lives (career and academics) they demonstrated a reluctance to do so in life-saving training programmes which would have also increased their life-quality. A probable reason for this can be that the individuals do not consider themselves susceptible to such life risks just because their peers do so.

\section{CONCLUSION}

By minute analysis of the results obtained from the above study conducted on a sample of 30 Indian adolescents, it can be concluded that a significant positive correlation exists between peer pressure and the self-reported tendency to utilise panic, evasiveness and complacency styles of decision making or decision coping patterns among adolescents. This study has been conducted on a limited sample which is based on a small geographical area. Therefore, we believe that adoption of amendments on certain points, such as utilizing a larger more comprehensive sample would provide more substantiated results with a greater potential for generalisation.

\section{AUTHOR INFORMATION}

The authors; Trisita Bhattacharyya (trisitab@gmail.com); Debasmita Roy (reemaroy013@gmail.com), Sunetra Chatterjee (sunetrachatterjee6@gmail.com) and Sharwat Aafreen Querashi (sharwataafreenquerashi@gmail.com) are $5^{\text {th }}$ semester Psychology Honours students from Asutosh College, University of Calcutta, Kolkata.

Dr. Tinni Dutta (tinnid@yahoo.com) is engaging herself as a faculty member in the Department of Psychology, Muralidhar Girls' College, Kolkata. She has provided keynote invited speech on various domains of psychology. She is a recipient of national and international awards. She has imparted her expertise and guidance in the completion of this study.

\section{REFERENCES}

[1] Acharya, A., \& OP, D. G. (2014). Influence of Peer Pressure On Brand Switching Among Indian College Students. International Journal of Current Research, 6(02), 5164-5171.

[2] Brown, B. B. (1982). The extent and effects of peer pressure among high school students: A retrospective analysis. Journal of youth and adolescence, 11(2), 121133.

[3] Brown, B. (1990). Peer Groups and Peer Cultures, In S. Feldman, and G. Elliott (eds.), At the Threshold: The Developing Adolescent. Cambridge, MA: Harvard University Press.

[4] Borsari, B., \& Carey, K. B. (2001). Peer influences on college drinking: A review of the research. Journal of substance abuse, 13(4), 391-424.

[5] Church, J., \& Stone, L. J. (1960). MOVING TOWARD MATURITY FROM INFANCY THROUGH ADOLESCENCE-THE EARLY SCHOOL YEARS, Children, 7, 113-114.

[6] Dacey, J. and Kenny, M. 1997. Adolescent development. USA: Mc Graw-Hill Inc.

[7] Elliot, A J. and Mc Gregor, H.A. 2001. A $2 \times 2$ achievement goal frame work. Journal of personality and social psychology, 80: 501-519.

[8] Friedman, I. A., \& Mann, L. (1993). Coping patterns in adolescent decision making: An Israeli-Australian comparison. Journal of adolescence, 16(2), 187-199.

[9] Garret, H. E. (1961). Statistics in Psychology and Education. Paragon International Publishers.

[10] Hurlock, E. B. (2001). Developmental psychology. Tata McGraw-Hill Education.

[11] Janis, I. L., \& Mann, L. (1977). Decision making: A psychological analysis of conflict, choice, and commitment. Free press.

[12] Kirchler, E., Palmonari, A. and Pombeni, M.L. 1993. Developmental tasks and adolescents' relationship with their peers and their family. In S. Jackson \& H. Rodrigue-Tome (Eds.). Adolescence and its social worlds. Hillsdale, NJ: Erlbaum.

[13] Lane, S. H. (2012). Decision-making behaviors for adolescent mothers enrolled in the nurse-family partnership. The University of North Carolina at Greensboro. 
[14] Mann, L., Harmoni, R., \& Power, C. (1989). Adolescent decision-making: The development of competence. Journal of adolescence, 12(3), 265-278.

[15] Newman, M. B., \& Newman, R. P. (2008). Through Life: A Psychosocial Approach. New York: Cengage Learning.

[16] Norman, G. (2010). Likert scales, levels of measurement and the "laws" of statistics. Advances in health sciences education, 15(5), 625-632.

[17] Palani, V., \& Mani, S. (2016). Exploratory Factor Analysis: Development of Perceived Peer Pressure Scale. International Journal of Information Science and Computing, 3(1), 31-41.

[18] Rathus, A.S. (2007). Childhood and Adolescence: Voyages in Development. New York: Cengage Learning.

[19] Tomé, G., de Matos, M. G., Simões, C., Camacho, I., \& AlvesDiniz, J. (2012). How can peer group influence the behavior of adolescents: explanatory model. Global journal of health science, 4(2), 26.

[20] Tuinstra, J., Van Sonderen, F. L. P., Groothoff, J. W., Van Den Heuvel, W. J. A., \& Post, D. (2000). Reliability, validity and structure of the Adolescent Decision Making Questionnaire among adolescents in The Netherlands. Personality and individual differences, 28(2), 273-285.

[21] UKEssays. (November 2018). Influence Of The Peer Group On The Adolescent Psychology Essay.

Retrieved from https://www.ukessays.com/essays/psychology/infl uence-of-the-peer-group-on-the-adolescentpsychology-essay.php?vref=1 